\title{
Nuclear modification factors, directed and elliptic flow of electrons from open heavy flavor decays in $\mathrm{Au}+\mathrm{Au}$ collisions from STAR
}

\author{
Matthew Kelsey for the STAR Collaboration ${ }^{\mathrm{a}}$ \\ ${ }^{a}$ Lawrence Berkeley National Laboratory, Berkeley, California 94720, USA
}

\begin{abstract}
We present the analyses of single electrons from semileptonic bottom and charm hadron decays at mid-rapidity in $\sqrt{s_{N N}}$ $=200$ and $54.4 \mathrm{GeV} \mathrm{Au}+\mathrm{Au}$ collisions (talk in Heavy-Flavor session III). The data at $\sqrt{s_{N N}}=200 \mathrm{GeV}$ incorporate information from the Heavy Flavor Tracker which enables the topological separation of electrons originating from bottom and charm hadron decays. We report the first STAR measurements at $\sqrt{s_{N N}}=200 \mathrm{GeV}$ of $v_{2}$ for bottom decay electrons as a function of $p_{\mathrm{T}}$ and $v_{1}$ for charm decay electrons as a function of electron rapidity. Additionally, we present the improved measurements of heavy-flavor decay electron $R_{A A}$ and a new measurement of the ratio of $R_{C P}$ between bottom and charm decay electrons. Finally, we also report the measurement of non-photonic electron $v_{2}$ in $\sqrt{s_{N N}}=54.4 \mathrm{GeV}$ data collected during the 2017 RHIC run.
\end{abstract}

Keywords: Heavy-Flavor, Electron, Quark, Anisotropic Flow, Parton Energy Loss, Nuclear Modification Factors

\section{Introduction}

Measurements of hadrons containing Heavy-Flavor (HF) quarks are one of the key probes of the Quark Gluon Plasma (QGP) produced in heavy-ion collisions since HF quark production is restricted to the initial hard scatterings before the formation of the QGP. Parton energy loss in the QGP medium is expected to follow a hierarchy ordered by parton color charge and mass, i.e., $\Delta E(g)>\Delta E(u, d)>\Delta E(c)>\Delta E(b)$. Measurements of charm hadron nuclear modification factors $\left(R_{A A}\right)$ at the RHIC $\left(\sqrt{s_{N N}}=200 \mathrm{GeV}\right)$ and LHC show values that are comparable to light-flavor hadrons at high transverse momentum $\left(p_{\mathrm{T}}\right)$. The similar values of $R_{A A}$ can be explained by models with parton energy loss in the QGP taking into account differences in fragmentation and spectra between light and charm quarks. A systematic comparison of bottom and charm hadron $R_{A A}$ can elucidate the mass dependence of parton energy loss since models predict significantly different values for both. These proceedings present measurements of single electron $R_{A A}$ from bottom and charm semileptonic decays using the Heavy Flavor Tracker (HFT) at STAR. Additionally, decay leptons preserve the HF hadron momentum direction and are therefore excellent proxies for anisotropic flow measurements which provide information of the HF quark transport properties of the QGP, and the initial tilt of the QGP bulk in the case of HF directed flow $\left(v_{1}\right)$. 


\section{Bottom and Charm Decay Electron Nuclear Modification Factors}

The fraction of bottom decay electrons to the sum of all non-photonic electrons (NPE) in Au+Au collisions is extracted with a template fit to the $\log (\mathrm{DCA} / \mathrm{cm})$ distribution, where the DCA is the $3 \mathrm{D}$ distance of closest approach of a candidate electron to the primary vertex, as shown in Fig. 1 left panel. The fractions of $N(b \rightarrow e) / N(b+c \rightarrow e)$ are shown in Fig. 1 middle and right panels for minimum bias (MB) and in bins of centrality, respectively, for $\mathrm{Au}+\mathrm{Au}$ collisions at $\sqrt{s_{N N}}=200 \mathrm{GeV}$. The ratios show a clear enhancement in MB and (mid-)central collisions with respect to the $\mathrm{p}+\mathrm{p}$ data ([1] and preliminary 2012 STAR data) and the FONLL predictions [2, 3]; The ratios in peripheral collisions are consistent with $p+p$ data and FONLL predictions.
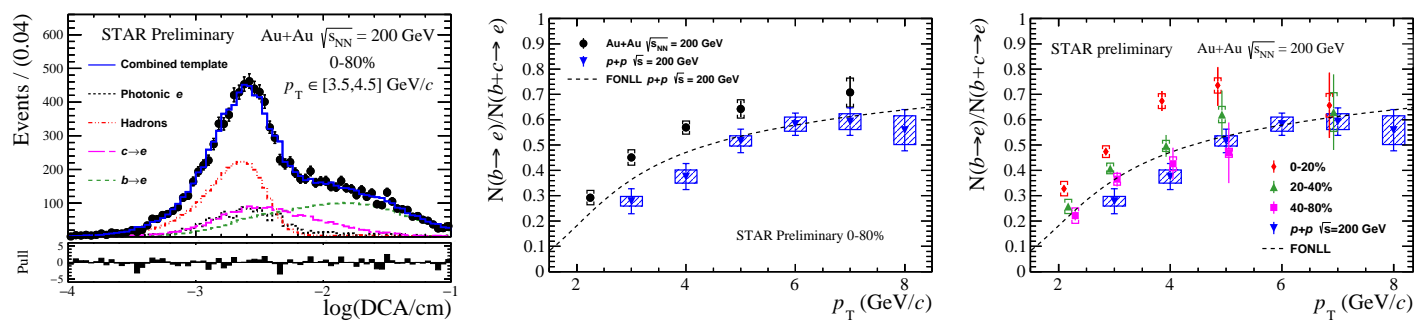

Fig. 1. Left: Example fit to the $\log (\mathrm{DCA} / \mathrm{cm})$ distribution in $\mathrm{MB} \sqrt{s_{N N}}=200 \mathrm{GeV} \mathrm{Au}+\mathrm{Au}$ collisions. Middle: The measured fraction of bottom decay electrons to all NPE electrons in MB $\sqrt{s_{N N}}=200 \mathrm{GeV} \mathrm{Au}+\mathrm{Au}$ collisions. Right: The bottom decay electron fraction as a function of $p_{T}$ in different collision centrality categories. In the middle and right panels the $\mathrm{p}+\mathrm{p}$ data ([1] and preliminary 2012 STAR data) and the FONLL predictions [2, 3] (black dashed line) are also shown. Statistical uncertainties are shown as error bars and systematic ones as brackets.

Using the bottom decay electron fractions in $\mathrm{Au}+\mathrm{Au}$ and $\mathrm{p}+\mathrm{p}$ collisions, and inclusive NPE $R_{A A}$ from preliminary STAR Au+Au data, the bottom and charm decay electron $R_{A A}$ are calculated and shown in Fig. 2 along with their ratio. The data show the bottom decay electron $R_{A A}$ is larger than that of charm decay electrons, and from a constant fit to the ratio this separation is $1.92 \pm 0.25$ (stat.) \pm 0.21 (syst.), which is significantly different from unity at roughly a $3 \sigma$ level. A null hypothesis for the ratio (blue shaded curve) is constructed by applying D meson $R_{A A}$ [5] to the $c / b \rightarrow e$ simulation, which takes into account the different decay kinematics in the $R_{A A}$ ratio. The p-value of the data to this curve is found to be .014 , disfavoring the hypothesis of identical charm and bottom hadron $R_{A A}$.

We compare to the Duke Langevin transport model [4] shown as the dotted lines in both panels, which contains the mass dependence of energy loss and other effects which may influence the measured $R_{A A}$ (e.g., hadronization and initial HF hadron $p_{\mathrm{T}}$ spectra). Within uncertainties the model is able to describe both the absolute values of $R_{A A}$ and their ratio. This compatibility between the data and model shows a good indication that bottom quarks lose less energy in the QGP compared to charm quarks.

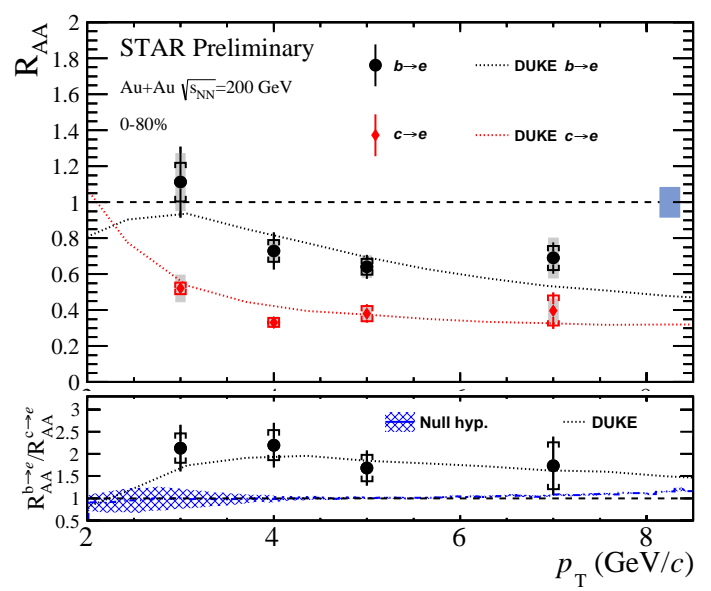

Fig. 2. The measured $R_{A A}$ for bottom and charm decay electrons as a function of electron $p_{\mathrm{T}}$. Statistical uncertainties on the data are shown as error bars and systematic ones as brackets; the shaded gray boxes show the common uncertainty due to the inclusive NPE $R_{A A}$ measurement. The blue shaded region at $R_{A A}=1$ shows the uncertainty on $\mathrm{N}_{\text {coll. }}$. The bottom panel shows the bottom to charm decay electron $R_{A A}$ ratio. The blue shaded curve shows the null hypothesis described in the text. In both panels Duke model [4] predictions are shown as the dotted lines. 
An additional measurement of the ratios of bottom to charm decay electron $R_{C P}$ is performed and the data show no strong $p_{\mathrm{T}}$ dependence. Performing the same constant fits described in the above text, we find $R_{C P}(0-20 \% / 40-80 \%)=1.68 \pm 0.15$ (stat.) \pm 0.12 (syst.) and $R_{C P}(0-20 \% / 20-40 \%)=1.38 \pm 0.08$ (stat.) \pm 0.03 (syst.). The significances of these measurements deviating from unity are $3.5 \sigma$ and $4.4 \sigma$, respectively.

\section{Bottom and Charm Decay Electron Anisotropic Flow}

The charm decay electron $v_{1}$ data are shown in Fig. 3 left and right panels for both charge averaged and split by charge, respectively. The average electron $\mathrm{d} v_{1} / \mathrm{d} y$ is in agreement with the STAR $D^{0}$ data [6], and is significant at a $5 \sigma$ level. To check for decorrelation in the $c \rightarrow e$ decay we folded the measured $D^{0} v_{1}$ into simulation, and find no significant $v_{1}$ information is lost in the decay electron within the measured $p_{\mathrm{T}}$ range. The better precision of the electron measurement will be able to further constrain the charm quark drag coefficient and tilt of the QGP bulk. The difference between $e^{+}$and $e^{-} v_{1}$ is compared to a hydrodynamic model including initial electromagnetic effects [7], and is consistent with zero within statistical uncertainties.
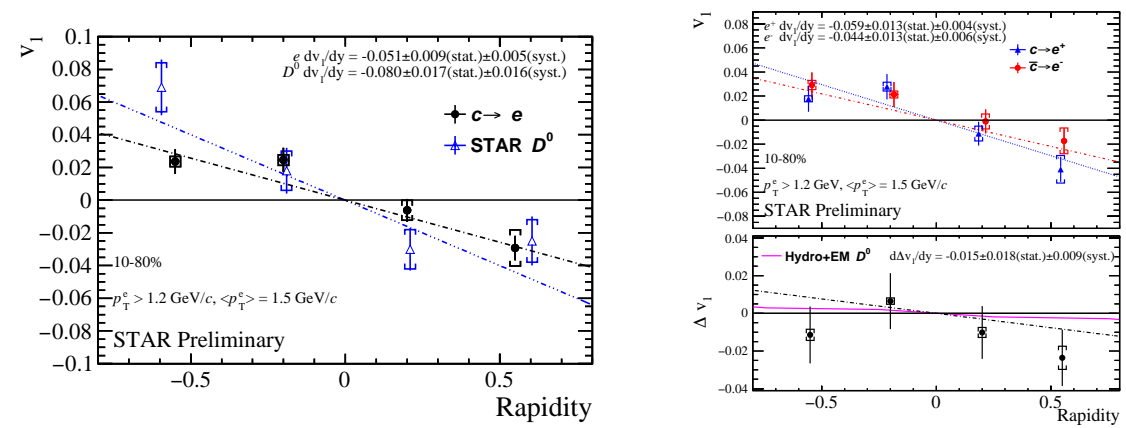

Fig. 3. Left: Measured data for the charm decay electron $v_{1}$ compared with the STAR $D^{0}$ measurement [6]. Linear fits to both the electron (black dot-dash) and $D^{0}$ (blue dot-dot-dot-dash) data are shown. Right: Measured $v_{1}$ split by electron charge are shown in the top panel with linear fits to the data shown as the blue dotted and red dot-dashed lines, respectively. The bottom panel shows the difference between $e^{+} v_{1}$ and $e^{-} v_{1}$ and a fit to the the difference as the black dot-dashed line, and a hydrodynamic model including initial electromagnetic effects [7] as the solid magenta curve. Statistical uncertainties are shown as error bars and systematic ones as brackets.

The charm and bottom decay electron $v_{2}$ data are shown in Fig. 4 left and right panels, respectively. The contributions from non-flow effects (shown as the shaded gray boxes) are estimated using electron-hadron correlations in semileptonic charm and bottom decays in PYTHIA. The measured charm decay electron $v_{2}$ is in agreement with the measured STAR $D^{0} v_{2}[8]$ folded to the decay electron in simulation shown as the shaded magenta band. For the bottom decay electron $v_{2}$ two event plane reconstruction methods are used with tracks reconstructed in the Time Projection Chamber (TPC, $-1<\eta<1$ ) or hits in the Forward Meson Spectrometer (FMS, $-2.5<\eta<-4$ ). In both panels the Duke model is also shown as the dotted black line, and within uncertainties is able to describe the $c \rightarrow e v_{2}$ data well. After subtracting non-flow contributions, the model is also able to describe the $b \rightarrow e v_{2}$ data within uncertainties. A null hypothesis $\left(v_{2}=0\right)$ for the bottom decay electron $v_{2}$ using the TPC event plane with the non-flow subtracted from the central value gives a p-value of $.00067(3.4 \sigma)$, indicating evidence for non-zero bottom decay electron $v_{2}$.

\section{Inclusive NPE $v_{2}$ in $\sqrt{s_{N N}}=54.4 \mathrm{GeV} \mathrm{Au}+\mathrm{Au}$ Collisions}

The inclusive NPE $v_{2}$ is measured in $\sqrt{s_{N N}}=54.4 \mathrm{GeV} \mathrm{Au}+\mathrm{Au}$ collisions in a similar way to previous STAR measurements at $\sqrt{s_{N N}}=200,62.4$, and $39 \mathrm{GeV}$ [9], of which the lower energy measurements were inconclusive due to the limited precision. The size of the data sample in this new measurement is roughly 15 times larger compared to the previous $62.4 \mathrm{GeV}$ measurement. The data are shown in Fig. 5 with the measurements at 200 and $62.4 \mathrm{GeV}$, and the $54.4 \mathrm{GeV}$ data show a significant inclusive NPE $v_{2}$ that is similar in magnitude to the data at $200 \mathrm{GeV}$. 

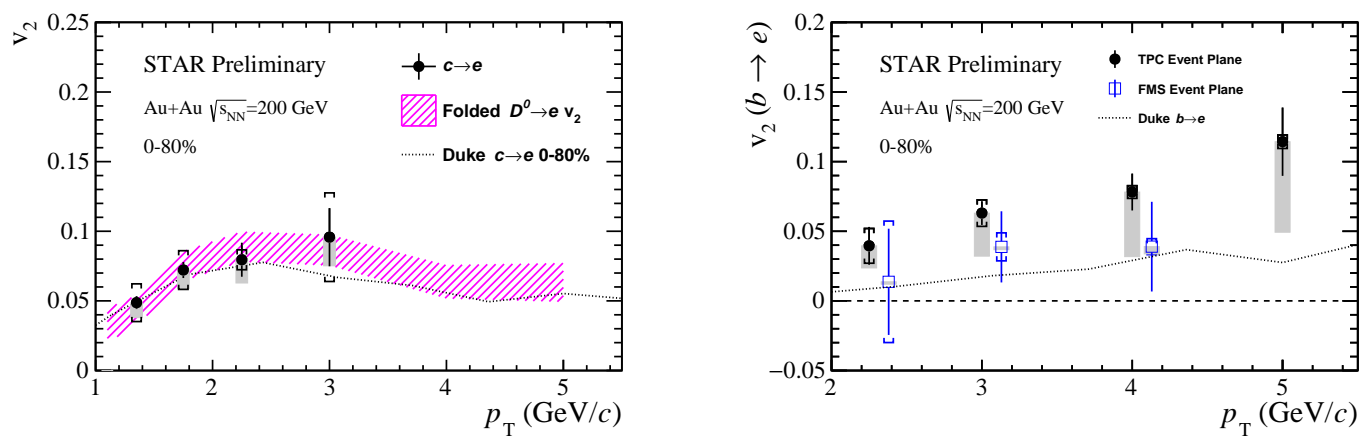

Fig. 4. Left: Measured data for the charm decay electron $v_{2}$. The folded STAR $D^{0}$ data $[8]$ are shown as the shaded magenta band (described in the text). Right: Measured bottom decay electron $v_{2}$ using the TPC (closed black circles) and FMS (open blue squares) event plane methods. Statistical uncertainties are shown as error bars and systematic ones as brackets; the gray boxes show the estimated non-flow contributions. In both panels the Duke model [4] is shown as the dotted black line.

\section{Conclusion}

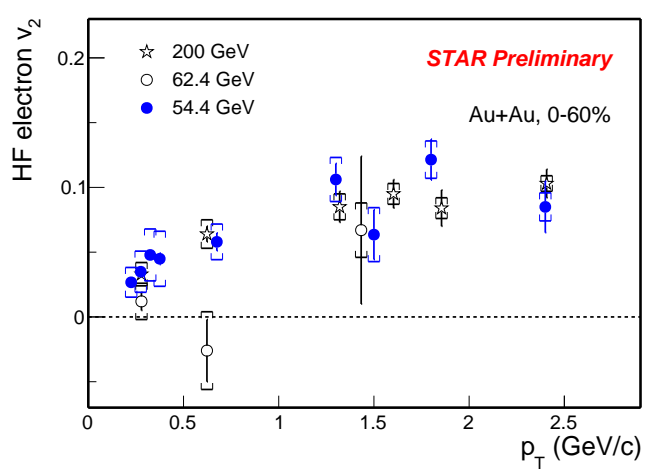

Fig. 5. Measured inclusive NPE $v_{2}$ in $\sqrt{s_{N N}}=54.4 \mathrm{GeV} \mathrm{Au}+\mathrm{Au}$ collisions. Also shown are the previous STAR measurements of inclusive NPE $v_{2}$ in $\sqrt{s_{N N}}=200$ and $62.4 \mathrm{GeV}$ Au+Au collisions [9]. Statistical uncertainties are shown as error bars and systematic ones as brackets. zero bottom decay electron $v_{2}(3.4 \sigma$ from zero)
at RHIC is presented. The measured $v_{2}$ values with the estimated non-flow contributions subtracted are consistent with Duke model calculations incorporating bottom quark transport in the QGP.

Finally, with the new data in $\sqrt{s_{N N}}=54.4 \mathrm{GeV} \mathrm{Au}+\mathrm{Au}$ collisions, we measure a significant inclusive NPE $v_{2}$ that is consistent with the previous STAR measurement at $\sqrt{s_{N N}}=200 \mathrm{GeV}$. This measurement indicates that charm quarks interact strongly with the QGP medium in $\sqrt{s_{N N}}=54.4 \mathrm{GeV} \mathrm{Au}+\mathrm{Au}$ collisions.

\section{References}

[1] M. M. Aggarwal, et al., Phys. Rev. Lett. 105 (2010) 202301.

[2] M. Cacciari, et al., JHEP 1210 (2012) 137.

[3] M. Cacciari, et al., arXiv:1507.06197.

[4] S. Cao, et al., Phys. Rev. C 92 (2015) 024907.

[5] J. Adam, et al., Phys. Rev. C 99 (2019) 034908.

[6] J. Adam, et al., Phys. Rev. Lett. 123 (2019) 162301.

[7] S. Chatterjee, P. BoÅek, Physics Letters B 798 (2019) 134955.

[8] L. Adamczyk, et al., Phys. Rev. Lett. 118 (2017) 212301.

[9] L. Adamczyk, et al., Phys. Rev. C 95 (2017) 034907.
We measure the charm and bottom decay electron $R_{A A}$ using the full STAR HFT data set and find that the bottom decay electron $R_{A A}$ is larger than the charm decay electron $R_{A A}$ by roughly a factor of two with a significance of about $3 \sigma$. The measured ratios of bottom and charm decay electron $R_{C P}$ show the same hierarchy with significance greater than $3.5 \sigma$. These observations, combined with the agreement with the Duke model including mass dependence of quark energy loss, are consistent with the mass hierarchy of parton energy loss.

The data for charm decay electron $v_{1}$ and $v_{2}$ are consistent with existing measurements of $D^{0}$ by STAR and provide additional constraints on QGP transport properties, and in the former case further information on the initial tilt of the QGP.

The first measurement of significant nonzero bottom decay electron $v_{2}(3.4 \sigma$ from zero $)$ 